\title{
Validation of a Physiologic Stability Index for Use in Critically Ill Infants and Children
}

\author{
TIMOTHY S. YEH, MURRAY M. POLLACK, ${ }^{(22)}$ URS E. RUTTIMANN, PETER R. HOLBROOK, \\ AND ALAN I. FIELDS \\ The Departments of Anesthesiology and Child Health and Development, Children's Hospital National Medical \\ Center and George Washington University School of Medicine, Washington, D.C., USA
}

\begin{abstract}
Summary
We developed a physiology-based scoring system, the Physiologic Stability Index (PSI) to assess severity of acute illness in the total population of pediatric Intensive Care Unit (ICU) patients. Thirty-four variables from seven physiologic systems were chosen, and the degree of abnormality of each variable was assigned a score reflecting the clinical importance of the derangements. Validity was demonstrated by comparing PSI to hospital mortality and to two other methods that reflect severity of illness, the Clinical Classification System (CCS) and the Therapeutic Intervention Scoring System (TISS). Four hundred and twentythree consecutive admissions to a multidisciplinary ICU were followed daily. Patients classified into higher CCS classes had significantly higher PSI scores $(P<\mathbf{0 . 0 0 1})$, and there was a highly significant correlation $(P<0.001)$ between PSI and TISS scores. The linear-logistic regression of observed mortality versus PSI was highly significant $(P<0.0001)$ and provided an excellent fit. Highly significant differences between survivors and nonsurvivors were observed for PSI scores $(P<0.001)$, as well as for composite slopes of the regression of PSI scores versus days of care $(P<0.001)$. These data demonstrate validity of the PSI scoring system.
\end{abstract}

\section{Abbreviations}

CCS, clinical classification system

ICU, intensive care unit

PSI, Physiologic Stability Index

TISS, Therapeutic Intervention Scoring System

Patient classification systems are useful for assessing severity of illness, care requirements, efficacy of therapy, and prognosis. In the ICU, a method of assessing severity of acute illness would be particularly desirable. Because patients in ICUs have great diversity of disease, classification systems that can be applied to all patients are most advantageous. Two such systems have gained wide acceptance. The CCS is a qualitative assessment of care requirements made on admission (4) and the TISS is a quantitative measure of amount of therapy (7). These systems have been used to assess adult $(4,8)$ and pediatric patients $(15$, $19)$ in ICUs. They are associated with mortality (8), severity of illness $(7,8,15,18,19)$, and cost (8).

A more direct method to assess severity of illness would be beneficial, especially in evaluating efficacy of therapy, interinstitutional comparisons of patient populations, and therapeutic techniques. CCS and TISS only indirectly reflect severity of illness by assessing therapeutic needs. A better method would directly assess severity of illness by quantitating physiologic stability. Most previous attempts at the development of systems based on physiologic variables have had drawbacks. Assessments that utilize specific variables of organ system dysfunction are limited to those particular disease processes only $(1,3,6,11,13)$ whereas systems that might have wider application require extensive, and invasive monitoring (16, 17). Knaus et al. (12) developed and validated a physiology-based classification system, APACHE, applicable to adults that makes use of routinely measured variables in the ICU and is designed to assess all major organ systems.

Thus far, no physiology-based classification system has been devised for use in the total population of infants and children in pediatric ICUs. The normal change in the physiology of the growing child has been one factor preventing the use in pediatric intensive care of any system using variable ranges derived for adults. We developed a physiology-based scoring system to assess severity of acute illness in the total population of infants and children admitted to the pediatric ICU. This system, the PSI, assesses severity of illness by quantitating the degree of derangement in seven major physiologic systems. By appropriately adjusting the ranges of some of the measured variables for different ages, all patients in pediatric ICU can be assessed. This study was conducted to validate the PSI.

\section{MATERIALS AND METHODS}

The PSI was developed by a group of pediatric intensivists. A list of variables and ranges of variables that met conditions defined below was developed and a consensus reached on all variables and their ranges. Within the context of the pediatric ICU, physiologic instability usually impacts through the respiratory, cardiovascular neurologic, or metabolic systems; therefore, these systems were most heavily weighed by the number and severity scores of the variables included. A total of 34 routinely or frequently measured variables from these seven systems was chosen.

After assessing the degree of abnormality, each variable was assigned a score of 1,3 , or 5 , which reflected the clinical importance of the derangement but not necessarily the amount of the deviation from the normal value. A score of 1 was assigned for a variable range that was sufficiently abnormal to cause concern but not necessarily mandate a change in therapy. A score of 3 was assigned to a variable range that was abnormal enough to cause significant concern and necessitate a change in therapy by most physicians in most clinical circumstances. A score of 5 was assigned for variable values indicating immediate life threatening situations. The PSI scoring system is shown in Table 1. Not all variables were deemed necessary to be adjusted for age, and not all warranted scores of 3 and 5 , because abnormalities of that variable taken alone might never be crucial enough to induce a change in therapy. The most abnormal value of a variable recorded within $24 \mathrm{~h}$ was used for scoring. The individual scores of the variables were added to obtain the final PSI score for each day. 
Table 1. Physiologic Stability Index (PSI)

\begin{tabular}{|c|c|c|c|}
\hline \multirow[b]{2}{*}{ System } & \multicolumn{3}{|c|}{ Points } \\
\hline & $1+$ & $3+$ & $5+$ \\
\hline \multicolumn{4}{|l|}{ Cardiovascular } \\
\hline \multicolumn{4}{|l|}{ Infants } \\
\hline \multirow[t]{2}{*}{ Blood pressure, systolic (torr) } & $55-65$ & $40-54$ & $<40$ \\
\hline & $130-160$ & $>160$ & \\
\hline \multirow[t]{2}{*}{ Heart rate (beat $/ \mathrm{min}$ ) } & $75-90$ & $50-74$ & $>220$ \\
\hline & $160-180$ & $181-220$ & $<50$ \\
\hline \multicolumn{4}{|l|}{ Children } \\
\hline Blood pressure, systolic (torr) & $\begin{array}{l}65-75 \\
150-200\end{array}$ & $\begin{array}{l}50-64 \\
>200\end{array}$ & $<50$ \\
\hline \multirow[t]{2}{*}{ Heart rate (beat/min) } & $60-80$ & $40-59$ & $>200$ \\
\hline & $150-170$ & $171-200$ & $<40$ \\
\hline \multicolumn{4}{|l|}{ All ages } \\
\hline Blood pressure, diastolic (torr) & $90-110$ & $>110$ & \\
\hline \multirow{3}{*}{$\begin{array}{l}\text { Cardiac index }\left[1 /\left(\min \cdot \mathrm{m}^{2}\right)\right] \\
\operatorname{avDO}_{2}(\operatorname{vol} \%)\end{array}$} & $2.0-3.0$ & $1.0-1.9$ & $<1.0$ \\
\hline & $<3.0$ & $>6.5$ & \\
\hline & $5.5-6.5$ & & \\
\hline CVP (torr) & $<0,>15$ & & \\
\hline PCWP/LA (torr) & $<5,15-25$ & $>25$ & \\
\hline \multicolumn{4}{|l|}{ Respiratory } \\
\hline \multicolumn{4}{|l|}{ Infants } \\
\hline Respiratory rate (breath/min) & $50-60$ & $61-90$ & $>90$ \\
\hline Children & & & \\
\hline Respiratory rate (breath/min) & $30-50$ & $51-70$ & $>70$ \\
\hline All ages & & & \\
\hline $\mathrm{PaO}_{2}$ (torr) & $50-60$ & $40-49$ & $<40$ \\
\hline $\mathrm{PaO}_{2} / \mathrm{FiO}_{2}$ & $200-300$ & $<200$ & \\
\hline $\mathrm{PaCO}_{2}$ (torr) & $<30,45-50$ & $51-65$ & $<65$ \\
\hline Neurologic & & & \\
\hline All ages & & & \\
\hline Glascow coma score & $8-11$ & $5-7$ & $<5$ \\
\hline Intracranial pressure (torr) & $15-20$ & $21-40$ & $>40$ \\
\hline Seizures & focal & grand $\mathrm{mal} / \mathrm{status}$ & \\
\hline Pupils & equal-sluggish & $\begin{array}{l}\text { unequal-dilated } \\
\text { sluggish }\end{array}$ & fixed/dilated \\
\hline Hematologic & & & \\
\hline All ages & & & \\
\hline Hemoglobin $(\mathrm{g} / \mathrm{dl})$ & $18.0-22.0$ & $3.0-5.0$ & $<3.0$ \\
\hline & $5.0-7.0$ & $22.1-25.0$ & $>25.0$ \\
\hline $\mathrm{WBC}\left(\right.$ cell $\left./ \mathrm{mm}^{3}\right)$ & $3-5,000$ & $<3,000$ & \\
\hline & $20-40,000$ & $>40,000$ & \\
\hline Platelets $\left(\right.$ cell $/ \mathrm{mm}^{3}$ ) & $20-50,000$ & $<20,000$ & \\
\hline & $>1$ million & & \\
\hline PT/PTT & $>1.5 \times$ control & & \\
\hline $\mathrm{FSP}(\mu \mathrm{g} / \mathrm{ml})$ & $>40$ & & \\
\hline Renal & & & \\
\hline All ages & & & \\
\hline BUN (mg/dl) & $40-100$ & $>100$ & \\
\hline Creatinine (mg/dl) & $2.0-10.0$ & $>10.0$ & \\
\hline Urine output $\left(\mathrm{cc} \cdot \mathrm{kg} \cdot \mathrm{h}^{-1}\right)$ & $0.5-1.0$ & $<0.5$ & \\
\hline Gastrointestinal & & & \\
\hline All ages & & & \\
\hline AST/ALT (IU/l) & $>100$ & & \\
\hline Amylase $(\mathrm{U} / \mathrm{l})$ & $>500$ & & \\
\hline Total bilirubin $(\mathrm{mg} / \mathrm{dl})$ & $>3.5$ & & \\
\hline Albumin (g/dl) & $1.2-2.0$ & $<1.2$ & \\
\hline Metabolic & & & \\
\hline All ages & & & \\
\hline Sodium (meq/l) & $115-125,150-160$ & $<115,>160$ & \\
\hline Potassium (meq/l) & $3.0-3.5$ & $2.5-2.9$ & $<2.5$ \\
\hline & $6.5-7.5$ & $7.6-8.0$ & $>8.0$ \\
\hline Calcium (mg/dl) & $7.0-8.0$ & $5.0-6.9$ & $<5.0$ \\
\hline & $12.0-15.0$ & $>15.0$ & \\
\hline Glucose (mg/dl) & $40-60$ & $20-39$ & $<20$ \\
\hline & $250-400$ & $>400$ & \\
\hline Osmolality (mosm/l) & $320-350$ & $>350$ & \\
\hline $\mathrm{pH}(\mathrm{U})$ & $7.20-7.30$ & $7.10-7.19$ & $<7.10$ \\
\hline & $7.55-7.65$ & $<7.65$ & \\
\hline $\mathrm{HCO}_{3}$ & $<16,>32$ & & \\
\hline
\end{tabular}


The validity of this system is contingent on the following assumptions: 1 ) all variables that need to be measured will be measured as indicated by patient care needs, 2) appropriate therapy will be given as indicated, 3) all measuring techniques are readily available to all institutions, and 4) severity of illness is a monotonic function of mutually independent and additive derangements observed in the separate physiologic systems. The latter assumption implies that the contribution from a single variable to overall severity of illness is independent of the disease process and the derangements of the other variables. The total severity of illness is a nondecreasing function of the sum of the scores of the individual variables.

In order to demonstrate the validity of this score, it was compared with observed mortality as well as to other methods that reflect severity of illness. The CCS and TISS were chosen as methods for comparison because of their applicability to all patients and their previously demonstrated applicability for use in the pediatric ICU population (19). The CCS is a qualitative assessment of severity of illness based on anticipated need of therapy. Class I patients are those requiring routine hospital care, Class II are those requiring prophylactic observation but who are physiologically stable, Class III are those who are physiologically stable and require intensive care nursing, and Class IV patients are physiologically unstable requiring frequent assessments and therapeutic changes by physicians and ICU nurses. TISS is a method of quantitating the amount of therapy by assigning points from 1-4 to various therapeutic interventions based on their complexity and invasiveness. For example, pulmonary artery catheters are given 4 points, intra-arterial catheters 3 points, central venous catheters 2 points, and hourly vital signs are given 1 point. The suggested additions of Silverman et al. (18) were included.

All medical and surgical admissions to a 16 bed multidisciplinary ICU were evaluated for a 7.5-mo period from December 1980 to July 1981 . These patients included infants and children, as well as neonates who had undergone cardiovascular surgery but not pediatric burns or premature newborns. At time of admission, patients were classified according to the CCS, diagnosis, age, and type of clinical service (medical, cardiovascular surgery, other surgery). CCS Class I patients were not admitted to the ICU.

Daily PSI and TISS scores were determined and means for all patients and for each CCS class were computed for the scores at the admission day, the average over the first $4 \mathrm{~d}$, the maximum score during the ICU stay, and the scores at the last day. The patients who were most ill, represented by Class IV patients, were examined in further detail by comparing the above scores for survivors and nonsurvivors. All but one death occurred in Class IV patients. Linear trends of successive daily PSI and TISS scores were computed by linear regression analysis for patients with a minimum of $3 \mathrm{~d}$ of care. Common slopes of these trends over the interval of the first $7 \mathrm{~d}$ in ICU were obtained from the individual slopes of the survivors and nonsurvivors separately by analysis of variance (2). The relationship between mortality and PSI scores was investigated in terms of a linear-logistic model (9). Statistical analysis for differences between means and between slopes of regression lines was conducted using the $t$ test.

\section{RESULTS}

Data were collected from a total of 423 patients. Ages ranged from $1 \mathrm{~d}$ to 254 mo with a median of 38 mo. Table 2 shows the
Table 2. Admitting service, $C C S^{*}$ class and age

\begin{tabular}{|c|c|c|c|c|}
\hline \multirow[b]{2}{*}{ Service } & \multicolumn{3}{|c|}{ Number } & \multirow{2}{*}{$\begin{array}{l}\text { Age (mo) } \\
\text { Median }\end{array}$} \\
\hline & $\begin{array}{c}\text { Class } \\
\text { II }\end{array}$ & $\begin{array}{c}\text { Class } \\
\text { III }\end{array}$ & $\begin{array}{c}\text { Class } \\
\text { IV } \\
\end{array}$ & \\
\hline \multicolumn{5}{|l|}{ Medicine } \\
\hline Survivors & 67 & 58 & 42 & 30 \\
\hline Nonsurvivors & 0 & 1 & 21 & 9.5 \\
\hline \multicolumn{5}{|c|}{ Cardiovascular surgery } \\
\hline Survivors & 2 & 34 & 56 & 24 \\
\hline Nonsurvivors & 0 & 0 & 11 & 6 \\
\hline \multicolumn{5}{|l|}{ Other surgery } \\
\hline Survivors & 60 & 45 & 21 & 92 \\
\hline Nonsurvivors & 0 & 1 & 5 & 25.5 \\
\hline \multicolumn{5}{|l|}{ Total } \\
\hline Survivors & 129 & 137 & 119 & 45 \\
\hline Nonsurvivors & 0 & 1 & 37 & 9 \\
\hline
\end{tabular}

* CCS, Clinical Classification System.

numbers, CCS classes, ages, and outcomes for the admitting services of medicine, cardiovascular surgery, and other surgery. Overall mortality was $9.0 \%(38 / 423)$ at 1 mo after admission to the ICU. Mortality was $0 \%$ for Class II patients, $0.7 \%$ for Class III patients, and $23.1 \%$ for Class IV patients. Admission, 4-d average, maximum, and last PSI and TISS scores were compared for different CCS groups. Figure 1 illustrates the PSI means and standard errors. The means of the three groups were statistically different $(P<0.001)$. Patients classified into higher CCS classes accumulated progressively higher PSI scores indicating an increasing degree of physiologic instability. The mean TISS score and standard errors are shown in Figure 2. Again, the means of the CCS groups were statistically different $(P<0.001)$, demonstrating that patients in the higher CCS groups received progressively more therapy (higher TISS scores). Actual values of mean PSI and TISS scores are given in Table 3 . There was also a highly significant correlation between TISS and PSI scores for all patients. This included admission scores $(r=0.546, P<0.001), 4$ d average scores $(r=0.604, P<0.001)$, maximum scores $(r=$ $0.640, P<0.001)$, and last scores $(r=0.665, P<0.001)$.

Figure 3 illustrates the relationship between 4 -d average PSI scores and hospital mortality. The bars represent the observed morality rates and the associated standard errors based on the patient numbers shown as insets. The curve shows the predicted mortality, $P$, attained by the logistic regression $P=\exp (0.277 \times$ PSI -5.241$) /[1+\exp (0.277 \times$ PSI -5.241$)]$. The coefficient of the PSI term was highly significant $(P<0.0001)$, indicating a strong association between hospital mortality and 4-d average PSI scores. The goodness of the fit is satisfactory with $\chi^{2} 30=$ $29.5, P<0.5$. Similar findings were obtained with respect to admission, maximum, and last scores. To investigate if the PSI score is influenced by age, the data base was separated by age $\leq$ $12 \mathrm{mo}$ or $>12 \mathrm{mo}$. For each age group, a stepwise multiple logistic regression of mortality versus 4 -d average PSI and age as regressor variables was performed. The results are summarized in Table 4. The regression coefficient for PSI are not statistically different for the two age groups $(P>0.43)$. Furthermore, age did not have any explanatory power for the prediction of mortality as indicated by the statistically insignificant regression coefficients in both age groups $(P>0.73, P>0.08)$.

Nonsurvivors also had consistently higher PSI and TISS scores

\footnotetext{
*Abbreviations: avDO $\mathrm{DO}_{2}=$ arterial minus mixed venous oxygen content difference; $\mathrm{CVP}$, central venous presure; PCWP, pulmonary capillary wedge pressure; LA, left arterial; PT, prothrombin time; PTT, partial thromboplastin time; FSP, fibrin split products; BUN, blood urea nitrogen; AST, aspartate aminotransferase; ALT, alanine aminotransferase, and $\mathrm{HCO}_{3}$, bicarbonate.

$\dagger$ Infant $\leq 12$ mo of age.

$\ddagger$ Children $>12$ mo of age.
} 


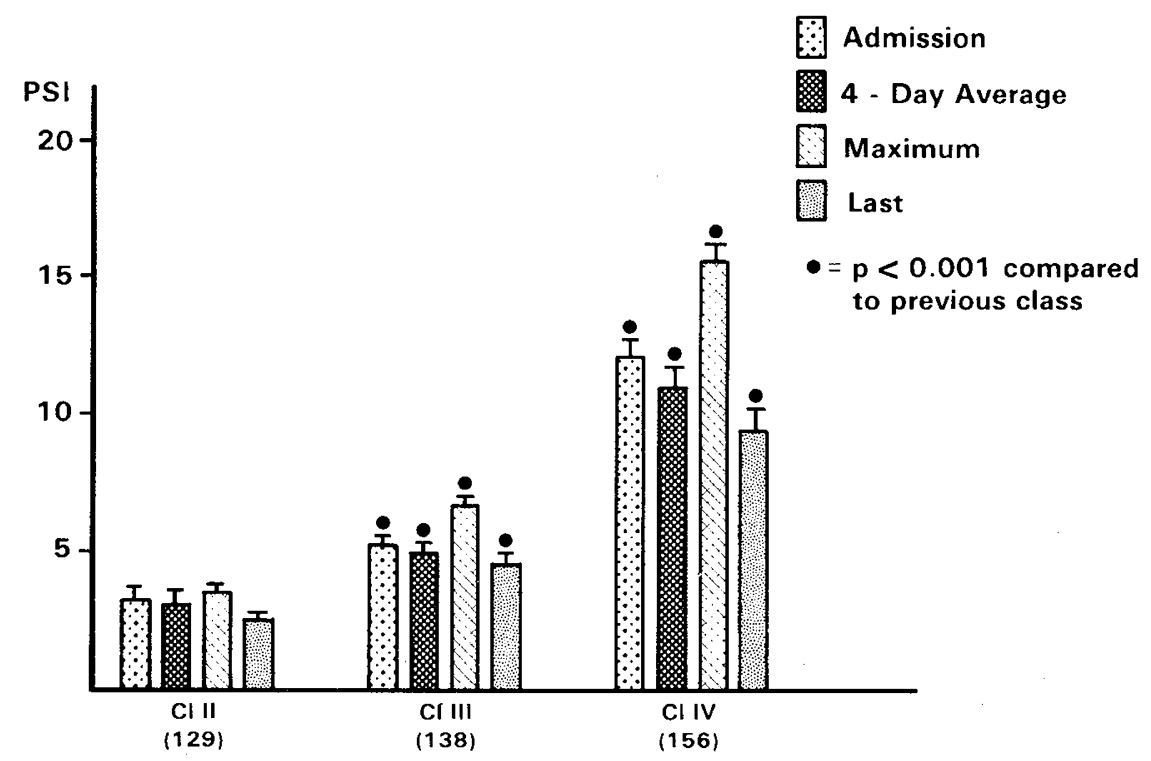

Fig. 1. Physiologic Stability Index (PSI) scores (mean \pm SEM) for Clinical Classification System classes.

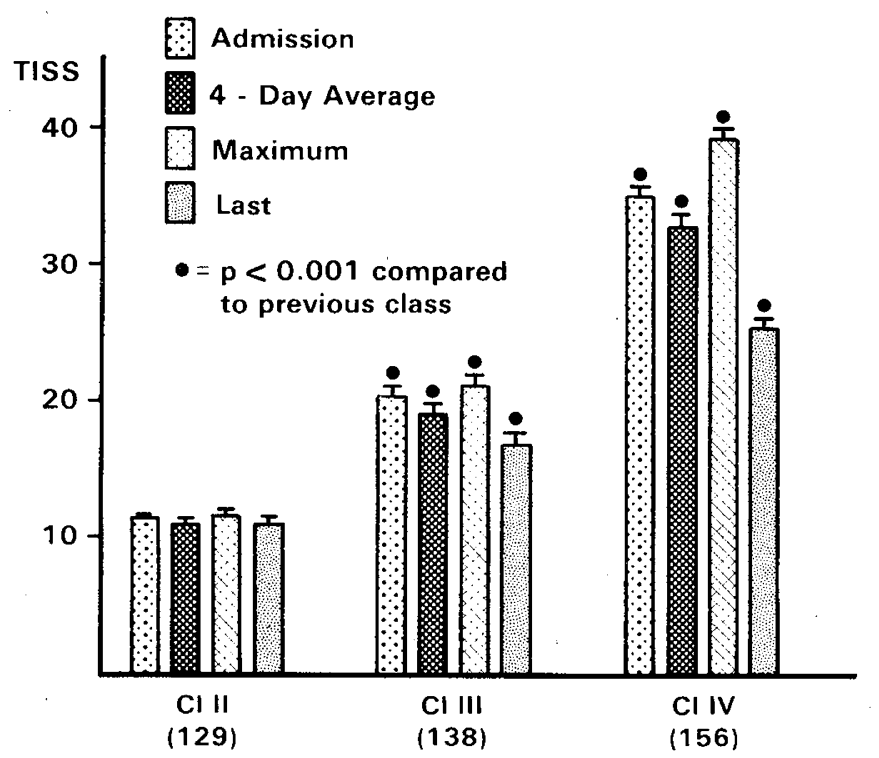

Fig. 2. Therapeutic Intervention Scoring System (TISS) scores (mean \pm SEM) for Clinical Classification System classes.

Table 3. Mean PSI and TISS values for CCS classes (mean \pm $S E M)^{*}$

\begin{tabular}{lrrrr}
\hline & Maximum & Admission & \multicolumn{1}{c}{$\begin{array}{c}4-\mathrm{d} \\
\text { average }\end{array}$} & Last day \\
\hline $\begin{array}{l}\text { Class II } \\
(n=129)\end{array}$ & & & & \\
$\quad$ PSI & $3.4 \pm 0.3$ & $3.1 \pm 0.3$ & $3.1 \pm 0.3$ & $2.8 \pm 0.2$ \\
$\quad$ TISS & $11.7 \pm 0.4$ & $11.4 \pm 0.4$ & $11.2 \pm 0.4$ & $11.1 \pm 0.4$ \\
Class III & & & & \\
$\quad(n=129)$ & & & & \\
$\quad$ PSI & $6.7 \pm 0.5$ & $5.2 \pm 0.4$ & $5.0 \pm 0.3$ & $4.7 \pm 0.4$ \\
$\quad$ TISS & $21.6 \pm 0.7$ & $20.3 \pm 0.7$ & $19.1 \pm 0.6$ & $16.7 \pm 0.6$ \\
Class IV & & & & \\
$\quad(n=129)$ & & & & \\
$\quad$ PSI & $15.7 \pm 0.8$ & $12.1 \pm 0.7$ & $11.0 \pm 0.6$ & $9.5 \pm 0.7$ \\
TISS & $39.0 \pm 0.9$ & $34.8 \pm 0.9$ & $32.7 \pm 0.8$ & $25.2 \pm 1.1$ \\
\hline
\end{tabular}

* Abbreviations: PSI, Physiologic Stability Index; TISS, Therapeutic Intervention Scoring System; and CCS, Clinical Classification System. when compared with survivors. Figure 4 illustrates this difference for the sickest group of patients, those in CCS Class IV. The mean PSI and TISS scores for admission, 4-d average, maximum, and last day were all significantly different for both PSI $(P<$ $0.001)$ and TISS $(P<0.001$, except for admission TISS score where $P<0.05$ ).

Composite linear trends of the PSI and TISS scores received during the first $7 \mathrm{~d}$ of ICU stay are shown in Figure 5 for Class II patients, and for Class III-IV survivors and nonsurvivors separately. The PSI trend for Class III-IV survivors had a negative slope of $-0.57 \mathrm{PSI}$ point/d, whereas the PSI slope for Class III-IV nonsurvivors was $+0.27 \mathrm{PSI}$ point/d. The TISS slope for Class III-IV survivors was $-1.81 \mathrm{TISS} \mathrm{pt} / \mathrm{d}$ versus +0.99 TISS point/d for the nonsurvivors in this group. Although the slopes of individual patients varied considerably, the differences of the composite slopes between the survivor and nonsurvivor groups were highly significant $(P<0.001)$ for both PSI and TISS. Only seven Class II patients remained in the ICU for three or more days and could be used to determine regression lines. The PSI slope for Class II patients was -0.03 PSI point/d and the TISS slope was +0.28 TISS point $/ \mathrm{d}$; both were not significantly different from zero ( $P=0.91$ for PSI, $P=0.57$ for TISS).

\section{DISCUSSION}

This study presents validation data on a new physiology-based patient classification system for pediatric intensive care patients. This system is designed to assess objectively the severity of acute illness. There exits a fundamental problem in validating any such system because the variable to be estimated, severity of illness, is unobservable. The outcome (survival, death) based on this unobservable variable is objectively measured. The unobservable variable may be assumed to be continuous with survival and death occurring at low and high values where the threshold value determining outcome is unknown. Clinical measurements are also observable and, when taken together in some unknown combination, define severity of illness.

Based on this conceptual model, there are formal statistical methods to estimate weights that should be given to each clinical measurement and which, when added together in a weighted sum, define severity of illness. The discriminant function approach $(1,11)$ has drawbacks in that 1 ) restricting assumptions for the distributions of the variables must be postulated, and 2) missing data values are not admissible. Distribution-free methods (14) eliminate the first restriction and permit a relaxation of the 


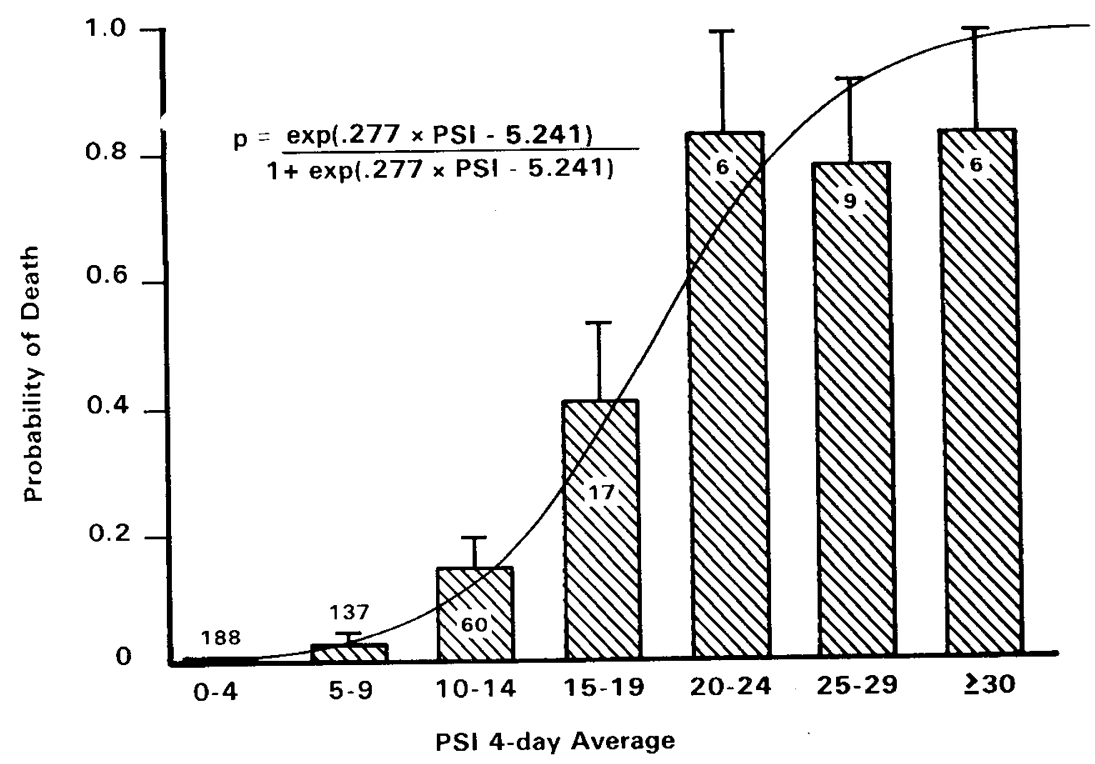

Fig. 3. Mortality vs Physiologic Stability Index (PSI). Curve shows predicted mortality based on linear-logistic regression analysis. Bars show observed mortality (mean \pm SEM) for PSI ranges based on the patient numbers indicated as insets. See text for further discussion.

Table 4. Estimated coefficients for the logistic regression of mortality $P^{*}$

\begin{tabular}{lcccc}
\hline & Constant & $\beta_{\mathrm{PSI}}$ & $\beta_{\text {age }}$ & $\chi^{2}$ of fit \\
\hline Age $\leq 12 \mathrm{mo}$ & -5.331 & $0.324 \ddagger$ & $<0.001$ & 37.53 \\
& $(5.755) \dagger$ & $(4.39)$ & $\ldots$ & $(\mathrm{df}=65)$ \\
& $P<0.0001$ & $P<0.0001$ & $P>0.73$ & $P=0.998$ \\
Age $>12 \mathrm{mo}$ & -5.332 & $0.257 \ddagger$ & -0.01 & 48.1 \\
& $(8.117)$ & $(5.77)$ & $(1.632)$ & $(\mathrm{df}=76)$ \\
& $P<0.0001$ & $P<0.0001$ & $P>0.08$ & $P=0.995$ \\
\hline
\end{tabular}

${ }^{*}$ Regression equation $\ln (P / 1-P)=$ constant $+\beta_{\mathrm{PSI}} \cdot \mathrm{PSI}+\beta_{\text {age }}$. age (mo)

$\dagger$ Asymptotic t-ratio.

$\ddagger$ Difference between $\beta_{\mathrm{PSI}}$ (age $\leq 12 \mathrm{mo}$ ) and $\beta_{\mathrm{PSI}}$ (age $>12 \mathrm{mo}$ ) is statistically not significant, $\mathrm{t}_{141}=0.798, P>0.43$.

second. Although the discriminant function approach is theoretically superior because it is multivariant, the distribution-free method is more practical, although some elements of subjectivity are introduced in the selection of ranges for the individual measurements (14) and a bias may be introduced if too many data are missing. Both methods require large patient samples to obtain unbiased estimates of the desired weights. This limits currently their applicability to pediatric intensive care because of the lack of sufficiently large patient samples.

Because of the difficulties in obtaining suitable numbers of patients required for these formal statistical methods, the approach of Knaus et al. (12) was followed. The derangements of the observed measurements were scored by employing the clinical judgment of pediatric intensivists. In terms of the conceptual model, the sum of these judgmental weights constitutes the unobservable variable which represents severity of illness. This approach retains the practical advantage of being distributionfree and explicitly permits "missing" data because, according to the assumptions outlined in "Materials and Methods," these data are assumed to be unnecessary for patient care and therefore "normal." The disadvantages of this approach are the subjectivity of the scores and the possibility of under or over measurements exists. The former drawback could be rectified by readjusting the scores within the framework of Bayesian statistics when a large data base exists.

Based on the conceptual model where the mortality rate is considered the observable variable and severity of illness is considered the unobservable variable, a formal method to test the adequacy of PSI to represent severity of illness is the linearlogistic regression of mortality versus PSI. The high significance of the regression coefficient for PSI in the logistic relation (Fig. 3 ) and the resulting index of the fit provide strong evidence for accepting PSI as a legitimate characterization of severity of illness. Furthermore, the comparison of survivors and nonsurvivors within the sickest group of patients (Fig. 4) clearly depicts large separation of scores for the two outcomes. Because of the difference in the scoring system for infants and children, separate logistic regressions of mortality versus PSI were performed for the two age groups. Age was also included as a regressor variable. The results indicate that the PSI score is equally valid for each age group; therefore, no age adjustment is required when the "goodness" of the scoring system is measured in terms of predicting hospital mortality.

Another method of validation is to compare a new system to previously developed classification systems. The authors have previously demonstrated that the CCS system is applicable to pediatric intensive care evaluation by demonstrating its association with mortality, duration of ICU stay, and TISS points (19). The strong association of PSI points to CCS classes (Fig. 1), its similar behavior to TISS points with respect to CCS classes (Fig. 2 ), and the significant correlation of PSI and TISS scores are confirmatory evidence of validation. The demonstration that the temporal change of patient stability as measured by PSI slopes acts in a manner consistent with clinical judgment (i.e., nonsurvivors become more unstable and survivors more stable) further adds validity to the PSI classification system. But it should be noted that the PSI slopes characterize groups of patients and cannot be used to predict the course of illness for individuals.

The PSI system was developed to aid the evaluation of severity of illness for acute disease. As such, it does not include a chronic health assessment as used in the system of Knaus et al. (12). A system that incorporates chronic health status might be beneficial in describing pediatric ICU patient populations, but would not seem to add further information to the evaluation of severity of acute illness. In fact, Knaus et al. (12) found that there was little benefit in adding the chronic health status evaluation to their acute physiology index for predicting short term outcome.

Both the PSI system and the APACHE system of Knaus et al. (12) represent severity of illness by assessment of physiologic variables. There is much similarity between the two system in the variables and abnormal ranges chosen. The major difference 


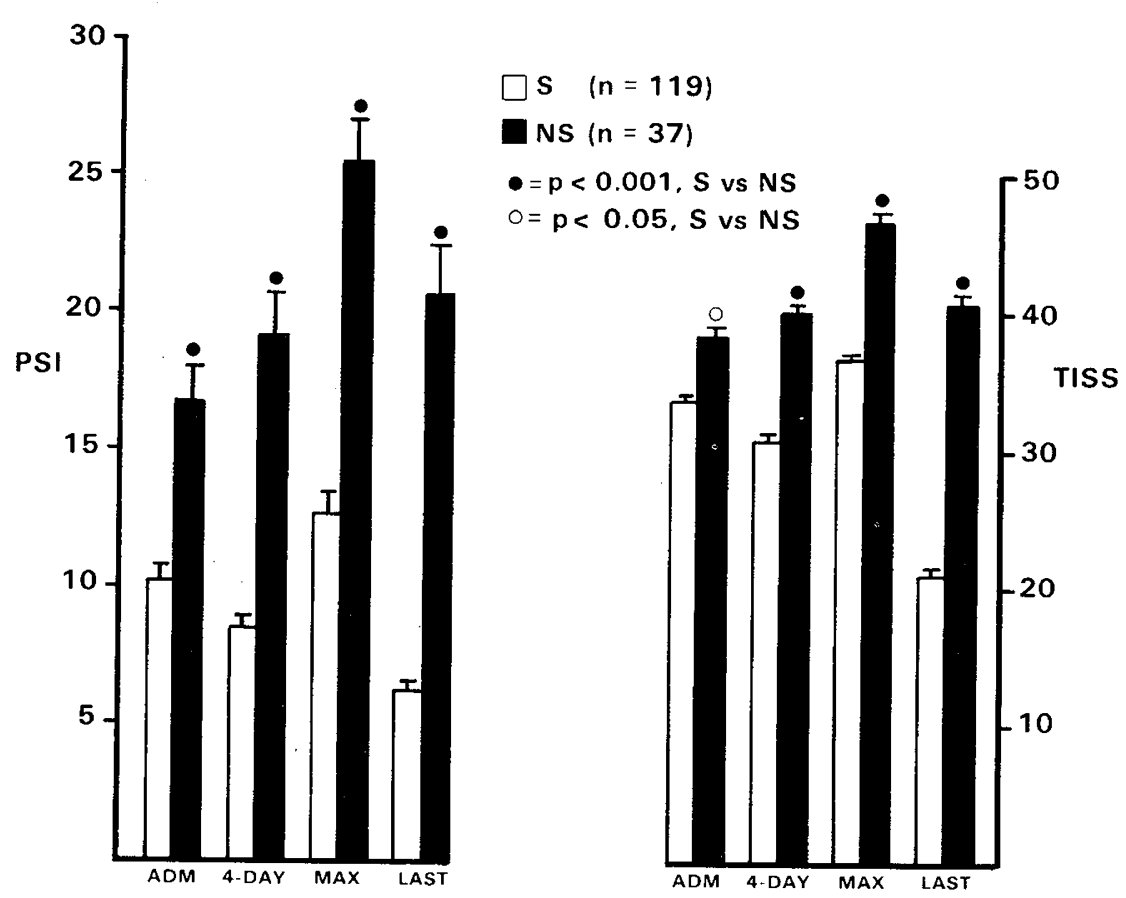

Fig. 4. Physiologic Stability Index (PSI) and Therapeutic Intervention Scoring System (TISS) scores (mean \pm SEM) for survivors (S) and nonsurvivors (NS) in CCS Class 4.

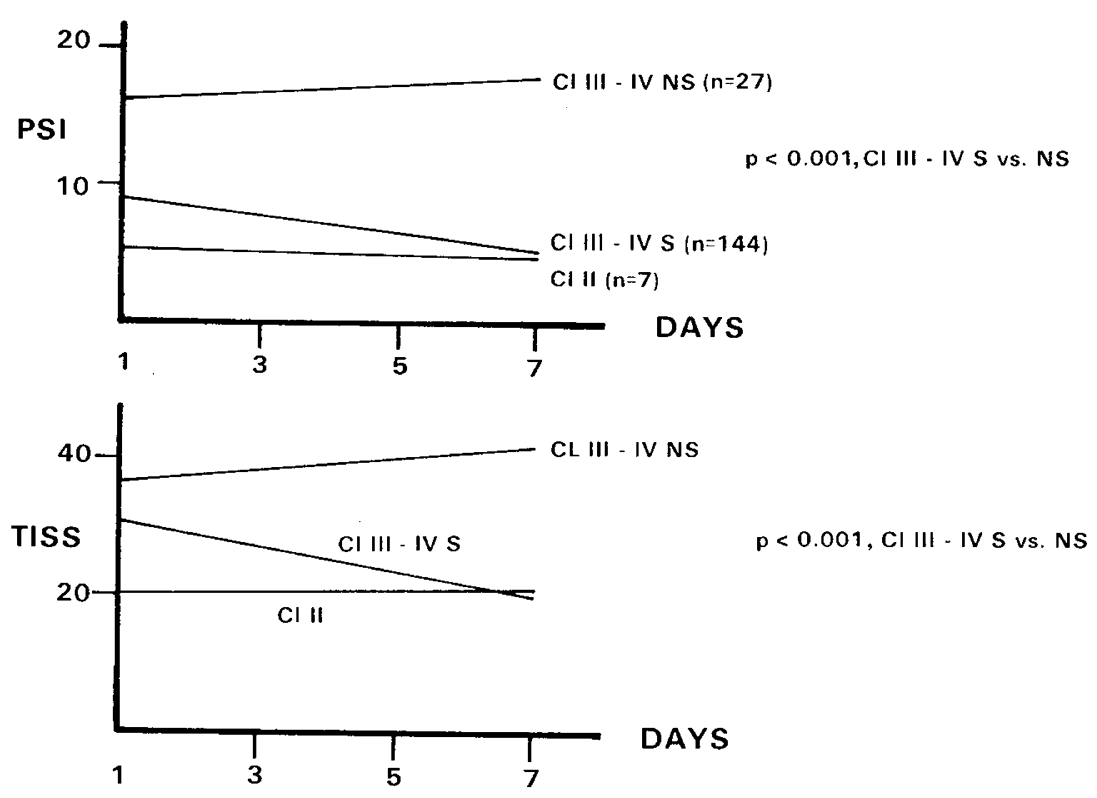

Fig. 5. Composite linear slopes of Physiologic Stability Index (PSI) and Therapeutic Intervention Scoring System (TISS) scores received during the first $7 \mathrm{~d}$ of intensive care unit stay for Clinical Classification System CCS Class (Cl) II, Class III-IV survivors (S) and Class III-IV nonsurvivors (NS).

between the two is the presence of age-adjusted variable ranges in the PSI system. Although both systems function well in the description of severity of illness, only the PSI is applicable to infants and children.

Further studies in evaluation of intensive care are necessary. Better methods of describing severity of illness are necessary for interinstitutional comparisons as well as for evaluating efficacy of therapy. For example, similar treatment protocols for identical disorders may have dramatically different results in different institutions $(5,10)$. A method of describing severity of illness might better identify patients who would benefit from more intensive therapy. Future evaluation of cost effectiveness of therapy and resource utilization will be necessary as pediatric intensive care becomes more developed. The PSI system is an empirically derived system based on the subjective, clinical judgments of pediatric intensivists. Its statistical validation is presented in this paper. Provided multi-institutional validation of the PSI system can be achieved, the true utility of this system will depend on its ability to help answer these questions.

\section{REFERENCES AND NOTES}

1. Afifi, A. A., Sacks, S. T., Lui, V. Y., Weil, M. H., and Shubin, H.: Accumulative prognostic index for patients with barbituarate glutethimide and meprobamate intoxication. N. Engl. J. Med., 285: 1497 (1971).

2. Armitage, P.: Statistical Methods in Medical Research. 4th ed., pp. 285-288 (John Wiley \& Sons, New York, 1977).

3. Bartlett, R. H., Gazzaniga, A. B., Wilson, A. F., Medley, T., and Wetmore, N.: Mortality prediction in adult respiratory insufficiency. Chest, 67:680 (1975). 
4. Civetta J. M. The inverse relationship between cost and survival. J. Surg. Res., 14: 265 (1973)

5. Cohn, J. N., Franciosa, J. A., Francis, G. S., Archibald, D., Tristani, F. Montero, A., Cintron, G., Clarke, J., Hager, D., Saunders, R., Cobb, F., Smith, R., Loeb, H., Settle, H. Effect of short-term infusion of sodium nitroprusside on mortality rate in acute myocardial infarction complicated by left ventricular failure. Results of a veterans cooperative study. N. Engl. J. Med, 306: 1129 (1982).

6. Cowley, R. A.: Sacco, W. J., Gill, W., Champion, H. R., Long, W. B., Goldfarb, M. A., and Sperrazza, J.: A prognostic index for severe trauma. J. Trauma, 14: 1029 (1974).

7. Cullen, D. J., Civetta, J. M., Briggs, B. A., and Ferrara, L. C.: Therapeutic intervention scoring system: a method for quantitative comparison of patient care. Crit. Care Med., 2: 57 (1974).

8. Cullen, D. J., Ferrara, L. C., Briggs, B. A., Walker, P. F., and Gilbert, J.: Survival hospitalization charges and follow-up results in critically ill patients. N. Eng. J. Med, 294: 982 (1976).

9. Dixon, W. J. and Brown, M. B.: BMPD-79, Biomedical Computer Programs. pp. 517.1-517.13. (University of California Press, Berkeley, 1979).

10. Durrer, J. D., Lie, K. I., Capelle, L. V., and Durrer, D.: Effect of sodium nitroprusside on mortality in acute myocardial infarction. N. Engl. J. Med., 306: 1121 (1982)

11. Hughes, W. L., Kalbfleisch, J. M., Brandt, E. N., and Costible, J. P.: Myocardial infarction: prognosis by discrimination analysis. Arch. Intern. Med., 111: 338 (1963).

12. Knaus, W. A., Zimmerman, J. E., Wagner, D. P., Draper, E. A. and Lawrence, D. E.: APACHE-acute physiology and chronic heaith evaluation: a physiologically based classification system. Crit. Care Med., 9: 591 (1981).

13. Norris, R. M., Brandt, P. W., Caughey, D. E., Lee, A. J., and Scott, P. J.: A new coronary prognostic index. Lancet, $1: 274$ (1969)

14. Pierchala, C. Shoemaker, W. C. Chang, P.: A comparison of some methods for early classification of survivors and nonsurvivors of postoperative shock. Comp. Biol. Med., 8: 279 (1978)

15. Rothstein, P. and Johnson, P.: Pediatric intensive care: factors that influence outcome. Crit. Care Med., 10: 34 (1982).

16. Shoemaker, W. C., Chang, P., Czer, L., et al.: Cardiorespiratory monitoring in postoperative patients. I. Predictor of outcome and severity of illness. Crit. Care Med., 7: 237 (1979).

17. Shoemaker, W. C. Pierchala, C., Chang, P., Bland, R., Czer, L., Shabot, M., and Clifton, J.F. Prediction of outcome and severity of illness by analysis of the frequency distributions of cardiorespiratory variables. Crit. Care Med., 5: 82 (1977).

18. Silverman, D. G., Goldiner, P. L., Kaye, B. A., Howland, W. S., Turnbill, A. D. The therapeutic intervention scoring system. An application to acutely ill cancer patients. Crit. Care Med., 3: 222 (1975).

19. Yeh, T. S., Pollack, M. M., Holbrook, P. R., Fields, A. I., and Ruttiman, U.: Assessment of pediatric intensive care-application of the therapeutic intervention scoring system. Crit. Care Med., 10: 497 (1982).

20. Presented at the American Pediatric Society and the Society for Pediatric Research Meetings, May, 1982.

21. The authors wish to acknowledge the assistance of Martha Wallace in the data collection and the encouragement of Drs. William Knaus and Douglas Wagner.

22. Requests for reprints should be addressed to: Murray M. Pollack, M.D. is: Associate Director, ICU, Children's Hospital National Medical Center, 111 Michigan Avenue, N.W., Washington, D.C. 20010

23. Received for publication November $22,1982$.

24. Accepted for publication July 19, 1983.

\title{
Ontogeny of the Immune System: Fetal Lamb as a Model
}

\author{
RUKMANI RAGHUNATHAN, ${ }^{(31)}$ MICHAEL E. MILLER, CARLA WUEST, AND JUDY FAUST \\ Department of Pediatrics, UCLA School of Medicine, Harbor-UCLA Medical Center, Torrance, California and \\ University of California, Davis School of Medicine, Sacramento, California, USA [M.E.M., C.W.]
}

\section{Summary}

We examined adult sheep lymphocytes for the following surface markers: Surface membrane immunoglobulin, antigen identified by anti-sheep thymocyte globulin and complement receptors. We quantitated sheep peripheral blood mononuclear leukocyte proliferative responses to mitogens and alloantigens, and demonstrated a neutrophil-directed chemotactic factor $(N$ LDCF) liberated by mitogen-stimulated sheep mononuclear cells. A comparison of adult and fetal sheep from 120-150 d of gestation demonstrated that 1 ) fetal sheep had adult proportions of surface membrane Ig+ and $\mathrm{T}+$ lymphocytes but a significant decrease in FcIgG receptor+ lymphocytes, 2) proliferative responses of fetal sheep blood mononuclear cells to mitogens and alloantigens were comparable to adult responses, and 3) fetal mononuclear leukocytes failed to produce the lymphokine, $\mathrm{N}$ LDCF, in response to mitogen stimulation.

\section{Abbreviations}

BSA, bovine serum albumin

CCE, counterflow centrifugation elutriation

CPM, counts/min

EA, antibody-coated sheep red cells
EAC, antibody- and complement-coated sheep red cells

FclgG, Fc portion of IgG

FITC, fluorescein isothiocyanate

FITC $F(a b)_{2}$ RxSIg, FITC conjugated $F(a b)_{2}$ fraction of rabbit anti-sheep Ig

GxRIg, goat anti-rabbit immunoglobulin

HBSS, Hanks balanced salt solution

LCM, lymphocyte culture medium

MFCS, McCoy's 5a medium with fetal calf serum

MHC, major histocompatibility locus

MLC, mixed leukocyte cultues

MNL, mononuclear leukocyte

N-LDCF, neutrophil-directed chemotactic factor

PMN, polymorphonuclear leukocytes

RAST, rabbit anti-sheep T-cell antibody

RITC, rhodamine isothiocyanate

RxSIg, rabbit anti-sheep immunoglobulin

SmIg, surface membrane immunoglobulin

Knowledge of the immune system's ontogeny is essential in understanding both the response of the fetus to intrauterine infections and other antigenic insults, and the effects of these 